Integrated Biologic and Clinical Approaches (The ABC Study) is an ongoing, investigator-initiated, clinical trial, conducted at the Oklahoma Medical Research Foundation (OMRF), with funding from Bristol Myers Squibb (NCT02270957).

\section{CS-06 STRUCTURAL BRAIN ABNORMALITIES IN YOUTH WITH SYSTEMIC LUPUS ERYTHEMATOSUS}

${ }^{1}$ Andrea Knight*, ${ }^{2}$ Michelle Vickery, ${ }^{3}$ Arastoo Vossough, ${ }^{4}$ Jimit Doshi, ${ }^{5}$ Guray Erus, ${ }^{6}$ Susan L Furth. 'Division of Rheumatology, Children's Hospital of Philadelphia; Perelman School of Medicine at the University of Pennsylvania, USA; ${ }^{2}$ Department of Pediatrics, Children's Hospital of Philadelphia, USA; ${ }^{3}$ Department of Radiology, Children's Hospital of Philadelphia; Perelman School of Medicine at the University of Pennsylvania, USA; ${ }^{4}$ Center for Biomedical Image Computing and Analytics, University of Pennsylvania, USA; ${ }^{5}$ Center for Biomedical Image Computing and Analytics, University of Pennsylvania, USA; ${ }^{6}$ Division of Nephrology, Children's Hospital of Philadelphia; Perelman School of Medicine at the University of Pennsylvania, USA

\subsection{6/lupus-2018-Ism.41}

Background Conventional magnetic resonance imaging (MRI) is limited for detection of clinically relevant brain changes in youth with systemic lupus erythematosus (SLE) and neuropsychiatric symptoms. We aimed to examine structural brain abnormalities in youth with SLE compared to healthy controls, utilizing advanced MRI analysis.

Methods We cross-sectionally compared images from clinicallyobtained brain MRI for adolescents and young adults with SLE, to those from age and sex-matched healthy control subjects with research MRI. Images were obtained from 20072015 on the same 3T scanner using a T1-weighted MPRAGE (magnetization-prepared rapid, acquisition gradient echo) protocol. A neuroradiologist performed conventional MRI reads, and was blinded to subsequent structural MRI volumetric analysis. An advanced multi-atlas segmentation algorithm divided the brain into 154 anatomical regions of interest (ROIs). Calculated volumes for large brain structures and ROIs were corrected for average intracranial volume of the sample, then compared between SLE and control subjects using univariate paired ttests, with Bonferroni correction for multiple comparisons.

Results 29 SLE were compared to 29 control images. SLE subjects had a median disease duration of 1.1 years (interquartile range, 0.3-3.3) at brain MRI, with clinical indications including cognitive changes (31\%), headache (24\%), depression/anxiety (21\%), and seizures (14\%). Nonspecific findings on conventional MRI read were higher for SLE subjects compared to controls, including T1 hyperintensities/T2 hypointensities $(48 \%$ vs $17 \%, \mathrm{p}<0.05)$ and mild diffuse volume loss ( $28 \%$ vs $0 \%, \mathrm{p}<0.01)$; hemorrhage/infarct was also higher $(14 \%$ vs $0 \%, \mathrm{p}<0.05)$. On structural MRI analysis, SLE subjects had significantly increased total white matter volume compared to controls $(\mathrm{p}<0.05)$, but no difference in total gray matter volume. For all subjects, total white and gray matter volumes were inversely correlated $(\rho=-0.86, \mathrm{p}<0.0001)$; age was correlated with increased total white matter $(\rho=0.49$, $\mathrm{p}<0.001)$ and decreased total gray matter volume $(\rho=-0.53$, $\mathrm{p}<0.001)$. Compared to controls, SLE subjects had significantly decreased volumes of 15 specific gray matter ROIs (figure 1) involved in decision-making, attention, memory, social cognition, emotional and language processing, and topographical and facial recognition. Sensitivity analyses excluding SLE subjects with clinician-diagnosed neuropsychiatric SLE $(n=6)$ did not change results of the structural MRI comparisons.

Conclusions Compared to healthy peers, youth with SLE early in their disease course have increased white matter and decreased regional gray matter volumes, possibly representing acceleration of normal age-related brain changes. Future study will examine the relationship between structural brain changes, disease-related factors and cognitive function in youth with SLE.

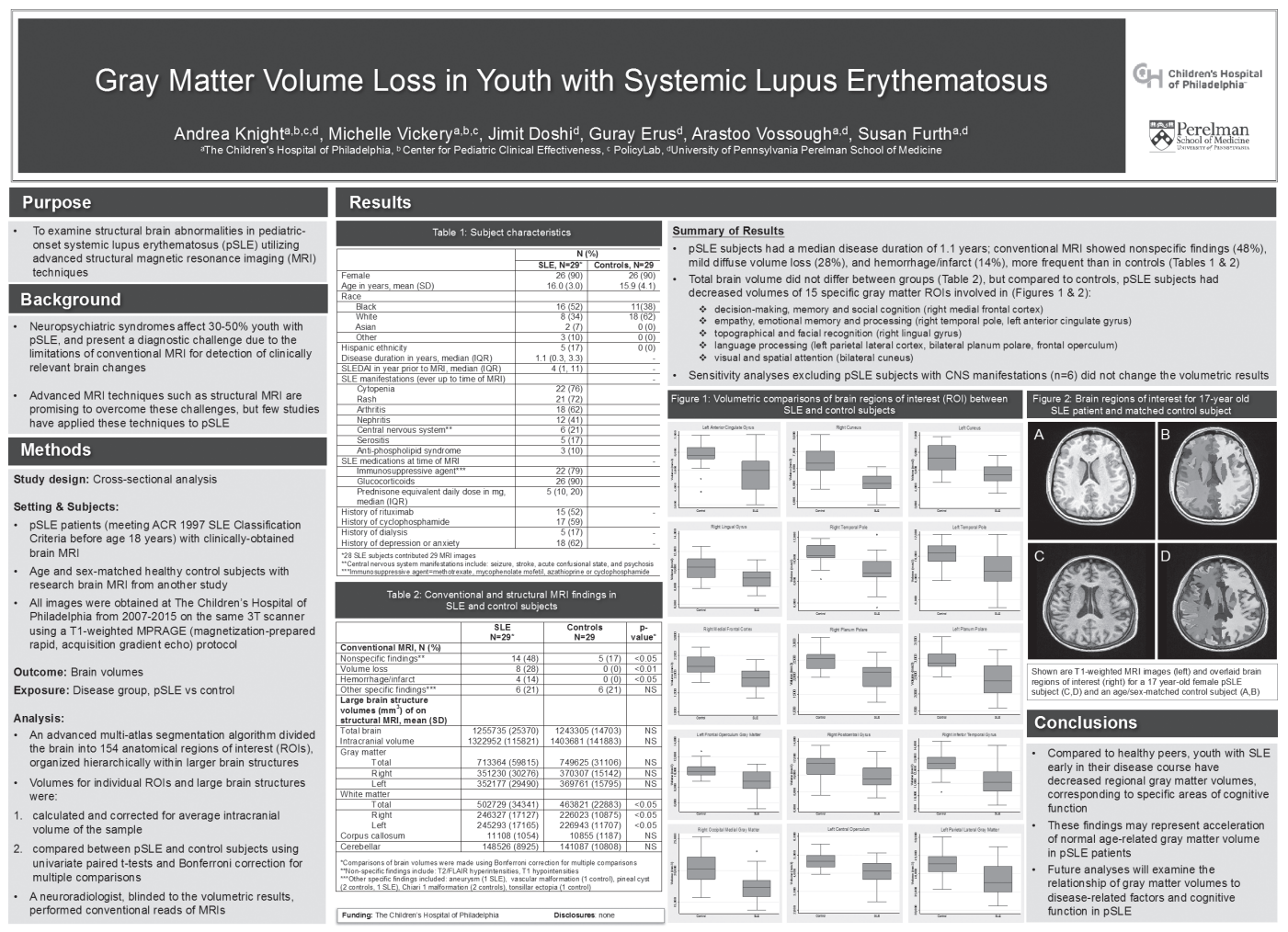

Abstract CS-06 Figure 1 Volumetric comparisons of gray matter regions of interest (ROI) between SLE and control subjects 